J o u r n a l of

Mathematics

and Applications

JMA No 42, pp 79-94 (2019)

\title{
A Generalization of the Hahn-Banach Theorem in Seminormed Quasilinear Spaces
}

\author{
Sümeyye Çakan and Yılmaz Yılmaz
}

AbSTRACT: The concept of normed quasilinear spaces which is a generalization of normed linear spaces gives us a new opportunity to study with a similar approach to classical functional analysis. In this study, we introduce the notion of seminormed quasilinear space as a generalization of normed quasilinear spaces and give various auxiliary results and examples. We present an analog of Hahn-Banach theorem, in seminormed quasilinear spaces.

AMS Subject Classification: 06B99, 32A70, 46A22, 46A99, 46B40, 47H04, 54F05.

Keywords and Phrases: Quasilinear spaces; Normed quasilinear spaces; Seminormed quasilinear spaces; Semimetrizable quasilinear spaces; Hahn-Banach theorem.

\section{Introduction}

Normed quasilinear spaces are introduced by Aseev, [2], in an effort to generalize normed linear spaces. A partial order relation was used to define normed quasilinear spaces. Motivated by [2], and using the framework and the tools given in [2], we developed the analysis in these spaces in $[5,6,7,8,9,12]$.

In this paper, we introduce the concept of seminormed quasilinear spaces and mention its some basic properties. Also we state and prove a version of Hahn-Banach theorem, one of the fundamental tools for the application of functional analysis, for seminormed quasilinear spaces. 


\section{Preliminaries and some results on quasilinear spaces and normed quasilinear spaces}

In this section, we present some basic definitions and results that appeared in [2] and [12] and which will be using in the sequel. Let us begin with Aseev's main definition.

Definition 2.1. [2] A set $X$ is called quasilinear space (qls, for short), if a partial order relation " $\preceq$ ", an algebraic sum operation and an operation of multiplication by real numbers are defined in it in such a way that the following conditions hold for any elements $x, y, z, v \in X$ and any $\alpha, \beta \in \mathbb{R}$ :

$$
\begin{gathered}
x \preceq x, \\
x \preceq z \text { if } x \preceq y \text { and } y \preceq z, \\
x=y \text { if } x \preceq y \text { and } y \preceq x, \\
x+y=y+x, \\
x+(y+z)=(x+y)+z,
\end{gathered}
$$

there exists an element $\theta \in X$ such that $x+\theta=x$,

$$
\begin{gathered}
\alpha \cdot(\beta \cdot x)=(\alpha \beta) \cdot x, \\
\alpha \cdot(x+y)=\alpha \cdot x+\alpha \cdot y, \\
1 \cdot x=x, \\
0 \cdot x=\theta, \\
(\alpha+\beta) \cdot x \preceq \alpha \cdot x+\beta \cdot x, \\
x+z \preceq y+v \text { if } x \preceq y \text { and } z \preceq v, \\
\alpha \cdot x \preceq \alpha \cdot y \text { if } x \preceq y .
\end{gathered}
$$

Generally, a qls $X$ with the partial order relation “ $\preceq$ " is denoted by $(X, \preceq)$. Here, we prefer denote the zero vector of $X$ by $\theta$ for clarity.

Every linear space is a qls with the partial order relation "=".

The most favorite example of qls which is not a linear space is the set of all nonempty, compact and convex subsets of real numbers with the inclusion relation " $\subseteq$ ", the algebraic sum operation

$$
A+B=\{a+b: a \in A, b \in B\}
$$

and multiplication operation by a real number $\lambda$ defined by

$$
\lambda \cdot A=\{\lambda a: a \in A\} .
$$


We denote this set by $\Omega_{C}(\mathbb{R})$.

Another one is $\Omega(\mathbb{R})$ which is the set of all nonempty compact subsets of real numbers.

In general, $\Omega(E)$ and $\Omega_{C}(E)$ stand for the space of all nonempty closed bounded and nonempty convex and closed bounded subsets of any normed linear space $E$, respectively. Both are nonlinear qls with the inclusion relation and a slight modification of addition operation by

$$
A+B=\overline{\{a+b: a \in A, b \in B\}}
$$

and multiplication operation by a $\lambda \in \mathbb{R}$ defined by $\lambda \cdot A=\{\lambda a: a \in A\}$. Where the closure is taken with respect to the standard topology in $\mathbb{R}$.

Lemma 2.1. [2] In a qls $(X, \preceq)$, the element $\theta$ is minimal, i.e., $x=\theta$ if $x \preceq \theta$.

Let $X$ be a qls and $Y \subseteq X$. Then $Y$ is called a subspace of $X$ if $Y$ is a qls with the same partial order relation and the restriction of the operations on $X$ to $Y$.

Theorem 2.1. [12] $Y$ is a subspace of qls $X$ if and only if $\alpha \cdot x+\beta \cdot y \in Y$ for every $x, y \in Y$ and $\alpha, \beta \in \mathbb{R}$.

An element $x^{\prime} \in X$ is called inverse of $x \in X$ if $x+x^{\prime}=\theta$. Further, if an inverse element exists, then it is unique. An element $x$ possessing inverse is called regular, otherwise is called singular. $X_{r}$ and $X_{s}$ stand for the sets of all regular and singular elements in $X$, respectively, [12].

It will be assumed throughout the text that $-x=(-1) \cdot x$.

Suppose that every element $x$ in a qls $X$ has inverse element $x^{\prime} \in X$. Then the partial order in $X$ is determined by equality, the distributivity condition in (2.11) holds and consequently, $X$ is a linear space, [2]. In a real linear space, "=" is only way to define a partial order such that the conditions (2.1)-(2.13) hold.

On the other hand, an element $x \in X$ is said to be symmetric if $-x=x$, and $X_{d}$ denotes the set of all symmetric elements.

$X_{r}, X_{d}$ and $X_{s} \cup\{\theta\}$ are subspaces of $X$ and called regular, symmetric and singular subspaces of $X$, respectively, [12].

Definition 2.2. [2] Let $(X, \preceq)$ be a qls. A real function $\|\cdot\|_{X}: X \longrightarrow \mathbb{R}$ is called a norm if the following conditions hold:

$$
\begin{gathered}
\|x\|_{X}>0 \text { if } x \neq \theta, \\
\|x+y\|_{X} \leq\|x\|_{X}+\|y\|_{X}, \\
\|\alpha \cdot x\|_{X}=|\alpha|\|x\|_{X}, \\
\text { if } x \preceq y, \text { then }\|x\|_{X} \leq\|y\|_{X},
\end{gathered}
$$

if for any $\varepsilon>0$ there exists an element $x_{\varepsilon} \in X$ such that

$$
x \preceq y+x_{\varepsilon} \text { and }\left\|x_{\varepsilon}\right\|_{X} \leq \varepsilon \text { then } x \preceq y .
$$

A qls $X$, with a norm defined on it, is called normed quasilinear space (normed qls, for short). 
Let $(X, \preceq)$ be a normed qls. Hausdorff metric or norm metric on $X$ is defined by the equality

$$
h_{X}(x, y)=\inf \left\{r \geq 0: x \preceq y+a_{1}^{r}, y \preceq x+a_{2}^{r} \text { and }\left\|a_{i}^{r}\right\| \leq r, i=1,2\right\} .
$$

Since $x \preceq y+(x-y)$ and $y \preceq x+(y-x)$ for any elements $x, y \in X$, the quantity $h_{X}(x, y)$ is well defined. Also, it is not hard to see that the function $h_{X}$ satisfies all of the metric axioms and we should note that $h_{X}(x, y)$ may not equal to $\|x-y\|_{X}$ if $X$ is a nonlinear qls; however $h_{X}(x, y) \leq\|x-y\|_{X}$ is always true for any elements $x, y \in X$. Therefore, we use the metric instead of the norm to discuss topological properties in normed quasilinear spaces. For example, $x_{n} \rightarrow x$ if and only if $h_{X}\left(x_{n}, x\right) \rightarrow 0$ for the sequence $\left(x_{n}\right)$ in a normed qls. Although, always $\left\|x_{n}-x\right\|_{X} \rightarrow 0$ implies $x_{n} \rightarrow x$ in normed quasilinear spaces, $x_{n} \rightarrow x$ may not imply $\left\|x_{n}-x\right\|_{X} \rightarrow 0$.

Let $E$ be a real normed linear space. Then $\Omega(E)$ and $\Omega_{C}(E)$ are normed quasilinear spaces with the norm defined by

$$
\|A\|_{\Omega}=\sup _{a \in A}\|a\|_{E} .
$$

In this case, the Hausdorff metric is defined as usual:

$$
h_{\Omega}(A, B)=\inf \{r \geq 0: A \subseteq B+S(\theta, r), B \subseteq A+S(\theta, r)\},
$$

where $S(\theta, r)$ is the closed ball of radius $r$ about $\theta \in X$, [2].

Lemma 2.2. [2] The operations of algebraic sum and multiplication by real numbers are continuous with respect to the Hausdorff metric. The norm is a continuous function with respect to the Hausdorff metric.

Lemma 2.3. [2] Let $X$ be a normed qls and $n$ be a positive integer.

a) Suppose that $x_{n} \rightarrow x_{0}, y_{n} \rightarrow y_{0}$ and $x_{n} \preceq y_{n}$ for any $n$. Then $x_{0} \preceq y_{0}$.

b) Let $x_{n} \rightarrow x_{0}$ and $z_{n} \rightarrow x_{0}$. If $x_{n} \preceq y_{n} \preceq z_{n}$ for any $n$, then $y_{n} \rightarrow x_{0}$.

c) If $x_{n}+y_{n} \rightarrow x_{0}$ and $y_{n} \rightarrow \theta$, then $x_{n} \rightarrow x_{0}$.

Definition 2.3. [2] Let $(X, \preceq)$ and $(Y, \preccurlyeq)$ be quasilinear spaces. A mapping $T: X \rightarrow$ $Y$ is called a quasilinear operator if it satisfies the following three conditions:

$$
\begin{gathered}
T(\alpha \cdot x)=\alpha \cdot T(x) \text { for any } \alpha \in \mathbb{R}, \\
T\left(x_{1}+x_{2}\right) \preccurlyeq T\left(x_{1}\right)+T\left(x_{2}\right), \\
\text { if } x_{1} \preceq x_{2}, \text { then } T\left(x_{1}\right) \preccurlyeq T\left(x_{2}\right) .
\end{gathered}
$$

If $X$ and $Y$ are linear spaces, then the definition of a quasilinear operator coincides with the usual definiton of linear operator. In this case, condition (2.22) is automatically satisfied. 
Definition 2.4. [2] Let $X$ and $Y$ be normed linear spaces. Any mapping from $X$ to $\Omega(Y)$ is called a multivalued mapping.

A quasilinear operator $T: X \rightarrow \Omega(Y)$ is called a multivalued quasilinear mapping. In this case, conditions (2.20) and (2.21) take the form

$$
\begin{gathered}
T(\alpha \cdot x)=\alpha \cdot T(x) \text { for any } \alpha \in \mathbb{R}, \\
T\left(x_{1}+x_{2}\right) \subset T\left(x_{1}\right)+T\left(x_{2}\right) .
\end{gathered}
$$

Also, condition (2.22) is automatically satisfied.

On the other hand, any quasilinear operator from $X$ to $\Omega(\mathbb{R})$ is called a quasilinear functional.

\section{Seminormed quasilinear spaces}

In this section, we propose a generalization of normed quasilinear spaces. Let us start the following definition.

Definition 3.1. Let $(X, \preceq)$ be a qls. A real function $p: X \rightarrow \mathbb{R}$ is called a seminorm if the following conditions hold:

$$
\begin{gathered}
p(x) \geq 0 \text { if } x \neq \theta, \\
p(x+y) \leq p(x)+p(y), \\
p(\alpha \cdot x)=|\alpha| p(x), \\
p(x) \leq p(y) \text { if } x \preceq y .
\end{gathered}
$$

A qls $X$ with a seminorm defined on it, is called seminormed quasilinear space (briefly, seminormed qls).

A seminorm $p$ is called total seminorm (or norm) if the condition

"if for any $\varepsilon>0$ there exists an element $x_{\varepsilon} \in X$ such that

$$
x \preceq y+x_{\epsilon} \text { and } p\left(x_{\epsilon}\right) \leq \epsilon \text { then } x \preceq y "
$$

holds.

Note that this definition is inspired from the definition of norm presented by Aseev in [2] and every seminormed (normed) qls is a semimetric (metric) qls.

Proposition 3.1. Let $(X, p)$ be a seminormed qls. Then the equality

$$
h_{X}(x, y)=\inf \left\{r \geq 0: x \preceq y+a_{1}^{r}, y \preceq x+a_{2}^{r}, p\left(a_{i}^{r}\right) \leq r, i=1,2\right\}
$$

defines a semimetric on $X$. If $p$ is total, $h_{X}$ becomes a metric. 
Proof. First of all, we should note that the quantity $h_{X}$ is well defined since $x \preceq$ $y+(x-y)$ and $y \preceq x+(y-x)$ for any elements $x, y \in X$.

Assume that $x=y$. Then $x \preceq y$ and $y \preceq x$. According to this,

$$
x \preceq y+a_{1}^{r} \text { and } y \preceq x+a_{2}^{r}
$$

for $a_{1}^{r}=a_{2}^{r}=\theta$. That implies $h_{X}(x, y)=0$ since $p\left(a_{1}^{r}\right)=p\left(a_{2}^{r}\right)=0$.

Clearly $h_{X}$ is symmetric. Further, remembering that

$$
h_{X}(x, z)=\inf \left\{r \geq 0: x \preceq z+a_{1}^{r}, z \preceq x+a_{2}^{r} \text { and } p\left(a_{i}^{r}\right) \leq \frac{r}{2}, i=1,2\right\}
$$

and

$$
h_{X}(z, y)=\inf \left\{r \geq 0: y \preceq z+b_{1}^{r}, z \preceq y+b_{2}^{r} \text { and } p\left(b_{i}^{r}\right) \leq \frac{r}{2}, i=1,2\right\},
$$

we write $x \preceq y+a_{1}^{r}+b_{2}^{r}$ for every elements $a_{1}^{r}$ and $b_{2}^{r}$ such that $x \preceq z+a_{1}^{r}$ and $z \preceq y+b_{2}^{r}$.

Similarly, we can say $y \preceq x+a_{2}^{r}+b_{1}^{r}$ for every elements $a_{2}^{r}$ and $b_{1}^{r}$ such that $y \preceq z+b_{1}^{r}$ and $z \preceq x+a_{2}^{r}$. Since

$$
p\left(a_{1}^{r}+b_{2}^{r}\right) \leq p\left(a_{1}^{r}\right)+p\left(b_{2}^{r}\right) \leq \frac{r}{2}+\frac{r}{2}=r
$$

and

$$
p\left(a_{2}^{r}+b_{1}^{r}\right) \leq p\left(a_{2}^{r}\right)+p\left(b_{1}^{r}\right) \leq \frac{r}{2}+\frac{r}{2}=r,
$$

we get $h_{X}(x, y) \leq h_{X}(x, z)+h_{X}(z, y)$. Because

$$
\begin{aligned}
h_{X}(x, y)= & \inf \left\{r \geq 0: x \preceq y+a_{1}^{r}+b_{2}^{r}, y \preceq x+a_{2}^{r}+b_{1}^{r},\right. \\
& \left.p\left(a_{1}^{r}+b_{2}^{r}\right) \leq r \text { and } p\left(a_{2}^{r}+b_{1}^{r}\right) \leq r\right\} \\
\leq & \inf \left\{r \geq 0: x \preceq z+a_{1}^{r}, z \preceq x+a_{2}^{r}, p\left(a_{i}^{r}\right) \leq \frac{r}{2}, i=1,2\right\} \\
+ & \inf \left\{r \geq 0: y \preceq z+b_{1}^{r}, z \preceq y+b_{2}^{r}, p\left(b_{i}^{r}\right) \leq \frac{r}{2}, i=1,2\right\} \\
= & h_{X}(x, z)+h_{X}(z, y) .
\end{aligned}
$$

Hence the equality (3.6) defines a semimetric.

Now let us show that $h_{X}$ becomes a metric whenever that the seminorm $p$ is total:

Let $p$ be total and $h_{X}(x, y)=0$. Then for any $\epsilon>0$ there exist elements $x_{1}^{\epsilon}, x_{2}^{\epsilon} \in X$ such that $x \preceq y+x_{1}^{\epsilon}, y \preceq x+x_{2}^{\epsilon}$ and $p\left(x_{i}^{\epsilon}\right) \leq \epsilon, i=1,2$. Hence the totality condition implies that $x \preceq y$ and $y \preceq x$, that is $x=y$.

The function $h_{X}$ defined with the equality in (3.6) is called semimetric (metric) derived from the seminorm (total seminorm) $p$.

Let $h_{X}$ be semimetric (metric) derived from the seminorm (total seminorm) $p$. Then the inequality $h_{X}(x, y) \leq p(x-y)$ holds for every $x, y \in X$. 
Proposition 3.2. Let $(X, p)$ be a seminormed qls and $h_{X}$ be semimetric (metric) derived from the seminorm (total seminorm) $p$. Then we have

i) $h_{X}(x+y, z+v) \leq h_{X}(x, z)+h_{X}(y, v)$,

ii) $h_{X}(\alpha \cdot x, \alpha \cdot y)=|\alpha| h_{X}(x, y)$

iii) $p(x)=h_{X}(x, \theta)$

for each $\alpha \in \mathbb{R}$ and every $x, y, z, v \in X$.

Proof. Let us show that the inequality $i$ ) holds. Taking into account the definition of $h_{X}$ and $\inf A+\inf B \geq \inf A+B$, and using (2.12), we write

$$
\begin{aligned}
& h_{X}(x, z)+h_{X}(y, v) \\
& =\inf \left\{r \geq 0: x \preceq z+a_{1}^{r}, z \preceq x+a_{2}^{r}, p\left(a_{i}^{r}\right) \leq r / 2, i=1,2\right\} \\
& +\inf \left\{r \geq 0: y \preceq v+b_{1}^{r}, v \preceq y+b_{2}^{r}, p\left(b_{i}^{r}\right) \leq r / 2, i=1,2\right\} \\
& \geq \inf \left\{\begin{array}{c}
r \geq 0: x \preceq z+a_{1}^{r}, y \preceq v+b_{1}^{r}, z \preceq x+a_{2}^{r}, v \preceq y+b_{2}^{r}, \\
p\left(a_{i}^{r}\right) \leq r / 2, p\left(b_{i}^{r}\right) \leq r / 2, i=1,2
\end{array}\right\} \\
& =\inf \left\{\begin{array}{c}
r \geq 0: x+y \preceq z+v+a_{1}^{r}+b_{1}^{r}, z+v \preceq x+y+a_{2}^{r}+b_{2}^{r}, \\
p\left(a_{i}^{r}+b_{i}^{r}\right) \leq r, i=1,2
\end{array}\right\} \\
& =h_{X}(x+y, z+v) .
\end{aligned}
$$

The equalities $i$ ) and $i i i$ ) can be also easily obtained.

Proposition 3.3. Let $(X, p)$ be a seminormed qls, $x, y \in X$ and $h_{X}$ be semimetric (metric) derived from the seminorm (total seminorm) $p$. Then

$$
h_{X}(x, \theta) \leq h_{X}(y, \theta) \text { if } x \preceq y .
$$

Further, quasilinear space operations are continuous with respect to the topology induced by $h_{X}$.

Proof. Primarily, we say that $x \preceq y$ implies $p(x) \leq p(y)$ since $p$ is seminorm. Considering

$$
p(x)=h_{X}(x, \theta) \text { and } p(y)=h_{X}(y, \theta),
$$

it is obtained $h_{X}(x, \theta) \leq h_{X}(y, \theta)$ whenever $x \preceq y$.

Since the topology derived from the semimetric $h_{X}$ is first countable topology, to say that addition and scalar multiplication operations are continuous, it will be sufficient to show that these operations are sequentially continuous.

For continuity of addition, let $\left(x_{n}\right)$ and $\left(y_{n}\right)$ be two sequences in $X$ such that $x_{n} \rightarrow x$ and $y_{n} \rightarrow y$. Then for every $\epsilon>0$ there exists $N \in \mathbb{N}$ such that

$$
x_{n} \preceq x+a_{1, n}^{\epsilon}, x \preceq x_{n}+a_{2, n}^{\epsilon} \text { and } p\left(a_{i, n}^{\epsilon}\right) \leq \frac{\epsilon}{2}, i=1,2
$$


and

$$
y_{n} \preceq y+b_{1, n}^{\epsilon}, y \preceq y_{n}+b_{2, n}^{\epsilon} \text { and } p\left(b_{i, n}^{\epsilon}\right) \leq \frac{\epsilon}{2}, i=1,2
$$

whenever $n \geq N$. Taking into account that $p$ is seminorm and using (2.12), we can write

$$
\begin{gathered}
x_{n}+y_{n} \preceq x+y+a_{1, n}^{\epsilon}+b_{1, n}^{\epsilon}, \\
x+y \preceq x_{n}+y_{n}+a_{2, n}^{\epsilon}+b_{2, n}^{\epsilon}
\end{gathered}
$$

and

$$
\begin{aligned}
& p\left(a_{1, n}^{\epsilon}+b_{1, n}^{\epsilon}\right) \leq p\left(a_{1, n}^{\epsilon}\right)+p\left(b_{1, n}^{\epsilon}\right) \leq \frac{\epsilon}{2}+\frac{\epsilon}{2}=\epsilon \\
& p\left(a_{2, n}^{\epsilon}+b_{2, n}^{\epsilon}\right) \leq p\left(a_{2, n}^{\epsilon}\right)+p\left(b_{2, n}^{\epsilon}\right) \leq \frac{\epsilon}{2}+\frac{\epsilon}{2}=\epsilon .
\end{aligned}
$$

These imply that $x_{n}+y_{n} \rightarrow x+y$.

Hence, it remains to show that multiplication operation is continuous. Let $\left(x_{n}\right)$ be a sequence in $X$ such that $x_{n} \rightarrow x$. Then for every $\epsilon>0$ there exists $N \in \mathbb{N}$ such that

$$
x_{n} \preceq x+a_{1, n}^{\epsilon}, x \preceq x_{n}+a_{2, n}^{\epsilon} \text { and } p\left(a_{i, n}^{\epsilon}\right) \leq \frac{\epsilon}{|\lambda|}, \lambda \in \mathbb{R}^{+}, i=1,2
$$

whenever $n \geq N$. Using (2.8), (2.12), (2.13) and the fact that $p$ is seminorm, we can say

$$
\begin{gathered}
\lambda \cdot x_{n} \preceq \lambda \cdot x+\lambda \cdot a_{1, n}^{\epsilon}, \\
\lambda \cdot x \preceq \lambda \cdot x_{n}+\lambda \cdot a_{2, n}^{\epsilon}
\end{gathered}
$$

and

$$
p\left(\lambda \cdot a_{i, n}^{\epsilon}\right) \leq|\lambda| p\left(a_{i, n}^{\epsilon}\right) \leq \epsilon, i=1,2 .
$$

This implies that $\lambda \cdot x_{n} \rightarrow \lambda \cdot x$.

Also, we note that the semimetric (metric) $h_{X}$ induced by a seminorm (total seminorm) on the qls $X$ is not translation invariant. But this semimetric (metric) satisfies the inequality

$$
h_{X}(x+a, y+a) \leq h_{X}(x, y), a \in X .
$$

Indeed,

$$
h_{X}(x+a, y+a) \leq h_{X}(x, y)+h_{X}(a, a)=h_{X}(x, y) .
$$

Now let us present an example of seminorm function which is not a norm.

Example 3.1. Consider the qls $\Omega_{C}\left(\mathbb{R}^{2}\right)$ and the function

$$
p(A)=\sup \left\{\left|x_{2}\right|:\left(x_{1}, x_{2}\right) \in A\right\}
$$

for any $A \in \Omega_{C}\left(\mathbb{R}^{2}\right)$. 
It is easy to see that $p$ holds seminorm axioms. On the other hand, $p$ is not a norm since $p(A)=0$, for element $A=\{(t, 0):-1 \leq t \leq 1\} \in \Omega_{C}\left(\mathbb{R}^{2}\right) \neq \theta$. Also the condition (2.18) is also not satisfied:

Let $A=\{(t, 0): 0 \leq t \leq 2\}, B=\{(t, 0): 0 \leq t \leq 1\}$ and $\epsilon>0$ be arbitrary. Let us define as

$$
A_{\epsilon}=\{(t+\epsilon, 0): 0 \leq t \leq 1\} .
$$

Then $p\left(A_{\epsilon}\right)=0$ and $A \subset B+A_{\epsilon}$, but $A \mp B$.

Example 3.2. The function $q(A)=\frac{p(A)}{1+p(A)}$ formed by aid of the seminorm $p$ in Example 3.1 is not a seminorm on $\Omega_{C}\left(\mathbb{R}^{2}\right)$, since

$$
q(\lambda \cdot A)=\frac{p(\lambda \cdot A)}{1+p(\lambda \cdot A)}=\frac{|\lambda| p(A)}{1+|\lambda| p(A)} \neq \lambda q(A) .
$$

In the following, we give an example of a semimetric map that is not a metric.

Example 3.3. Let $A, B \in \Omega_{C}\left(\mathbb{R}^{2}\right)$ and

$$
d(A, B)=\sup \left\{\sqrt{\left|a_{1}-b_{1}\right|}:\left(a_{1}, a_{2}\right) \in A,\left(b_{1}, b_{2}\right) \in B\right\} .
$$

Firstly let us show that this formula defines a function from $\Omega_{C}\left(\mathbb{R}^{2}\right)$ to $\mathbb{R}$ :

Consider the projection

$$
p_{1}: \mathbb{R}^{2} \rightarrow \mathbb{R}, p_{1}\left(a_{1}, a_{2}\right)=a_{1}
$$

and remember that $p_{1}$ is continuous. $p_{1}(A)$ and $p_{1}(B)$ are compact subsets of $\mathbb{R}$ since $A$ and $B$ are compact in $\mathbb{R}^{2}$. Hence there exist the numbers $M_{1}, M_{2} \geq 0$ such that $|x| \leq M_{1}$ for every $x \in p_{1}(A)$ and $|x| \leq M_{2}$ for every $x \in p_{1}(B)$. Therefore, since

$$
\begin{aligned}
& \sup \left\{\sqrt{\left|a_{1}-b_{1}\right|}:\left(a_{1}, a_{2}\right) \in A,\left(b_{1}, b_{2}\right) \in B\right\} \\
& =\sup \left\{\sqrt{\left|a_{1}-b_{1}\right|}: a_{1} \in p_{1}(A), b_{1} \in p_{1}(B)\right\}
\end{aligned}
$$

and $|x| \leq \sqrt{M_{1}+M_{2}}$ for $x \in\left\{\sqrt{\left|a_{1}-b_{1}\right|}: a_{1} \in p_{1}(A), b_{1} \in p_{1}(B)\right\}$, the function $d$ is well defined.

It is easy to verify that $d$ is a semimetric. But $d$ is not a metric on $\Omega_{C}\left(\mathbb{R}^{2}\right)$. Indeed, for elements $A=\{(2,3)\}$ and $B=\{(2,4)\}$ in $\Omega_{C}\left(\mathbb{R}^{2}\right), d(A, B)=0$, but $A \neq B$.

On the other hand, we can show that the semimetric $d$ defined with (3.8) holds the condition (3.7) and the algebraic operations on $\Omega_{C}\left(\mathbb{R}^{2}\right)$ are continuous according to this semimetric.

For continuity of addition, let $\left(A_{n}\right)$ and $\left(B_{n}\right)$ be sequences in $\Omega_{C}\left(\mathbb{R}^{2}\right)$ such that $A_{n} \rightarrow A, B_{n} \rightarrow B$ and we take any $x_{n} \in A_{n}+B_{n}$. Then there exist $a_{n} \in A_{n}$ and $b_{n} \in B_{n}$ such that $x_{n}=a_{n}+b_{n}$. We can write as $a_{n}=\left(a_{1, n}, a_{2, n}\right)$ and $b_{n}=\left(b_{1, n}, b_{2, n}\right)$ since $A_{n}, B_{n} \in \Omega_{C}\left(\mathbb{R}^{2}\right)$. Because of $A_{n} \rightarrow A, B_{n} \rightarrow B$, we have

$$
d\left(A_{n}, A\right)=\sup \left\{\sqrt{\left|a_{1, n}-a_{1}\right|}:\left(a_{1, n}, a_{2, n}\right) \in A_{n},\left(a_{1}, a_{2}\right) \in A\right\} \rightarrow 0
$$


and

$$
d\left(B_{n}, B\right)=\sup \left\{\sqrt{\left|b_{1, n}-b_{1}\right|}:\left(b_{1, n}, b_{2, n}\right) \in B_{n},\left(b_{1}, b_{2}\right) \in B\right\} \rightarrow 0,
$$

whenever $n \rightarrow \infty$. Hence, we obtain that

$$
\begin{aligned}
& d\left(A_{n}+B_{n}, A+B\right) \\
& =\sup \left\{\sqrt{\left|a_{1, n}+b_{1, n}-\left(a_{1}+b_{1}\right)\right|}:\left(a_{1, n}+b_{1, n}, a_{2, n}+b_{2, n}\right) \in A_{n}+B_{n},\right. \\
& \qquad \sup \left\{\sqrt{\left|a_{1}+b_{1}, a_{2}+a_{1}\right|}:\left(a_{1, n}, a_{2, n}\right) \in A+B\right\} \\
& +\sup \left\{\sqrt{\left|b_{1, n}-b_{1}\right|}:\left(b_{1, n}, b_{2, n}\right) \in B_{n},\left(a_{1}, a_{2}, b_{2}\right) \in B\right\} \\
& \rightarrow 0+0=0(n \rightarrow \infty) .
\end{aligned}
$$

This shows that the addition operation is continuous. Similarly it can be seen that the real-scalar multiplication operation is continuous. Then

Lastly it remains to show that the condition (3.7) is satisfied. Assume that $A \subseteq B$.

$$
\begin{aligned}
d(A, \theta) & =\sup \left\{\sqrt{\left|a_{1}\right|}:\left(a_{1}, a_{2}\right) \in A\right\} \\
& \leq \sup \left\{\sqrt{\left|a_{1}\right|}:\left(a_{1}, a_{2}\right) \in B\right\} \\
& =d(B, \theta) .
\end{aligned}
$$

Remark 3.1. Every semimetric on a qls may not be obtained from a seminorm. In Example 3.3, if the semimetric defined on $\Omega_{C}\left(\mathbb{R}^{2}\right)$ is obtained from a seminorm, the property ii) in Proposition 3.2 should hold. However, we see that

$$
\begin{aligned}
d(\lambda \cdot A, \lambda \cdot B) & =\sup \left\{\sqrt{\left|\lambda \cdot a_{1}-\lambda \cdot b_{1}\right|}:\left(\lambda \cdot a_{1}, \lambda \cdot a_{2}\right) \in \lambda \cdot A,\left(\lambda \cdot b_{1}, \lambda \cdot b_{2}\right) \in \lambda \cdot B\right\} \\
& =\sqrt{|\lambda|} \sup \left\{\sqrt{\left|a_{1}-b_{1}\right|}:\left(a_{1}, a_{2}\right) \in A,\left(b_{1}, b_{2}\right) \in B\right\} \\
& =\sqrt{|\lambda|} d(A, B) .
\end{aligned}
$$

The following proposition is a comment of the condition (2.18).

Proposition 3.4. Let $X$ be a normed qls, $\mathcal{N}_{\theta}$ is the family of all neighbourhoods of $\theta$ and $x, y \in X$. If for any $V \in \mathcal{N}_{\theta}$ there exists some $b \in V$ such that $x \preceq y+b$, then $x \preceq y$.

Remark 3.2. In Proposition 3.4, the hypothesis "Let $\mathrm{X}$ be a normed qls" is indispensable. Indeed, Let us recall from Example 3.1 that the function

$$
p(A)=\sup \left\{\left|x_{2}\right|:\left(x_{1}, x_{2}\right) \in A\right\}, A \in \Omega_{C}\left(\mathbb{R}^{2}\right)
$$


is seminorm on $\Omega_{C}\left(\mathbb{R}^{2}\right)$. We can construct a topology $\tau$ on $\Omega_{C}\left(\mathbb{R}^{2}\right)$ by aid of $p$ in such a way that

$$
U \in \tau \Leftrightarrow\{A: p(A)<\epsilon\} \subseteq U, \text { for some } \epsilon>0 .
$$

We note that $\tau$ is a semimetrizable topology with the semimetric

$$
d(A, B)=\inf \left\{r \geq 0: A \subseteq B+C_{1}^{r}, B \subseteq A+C_{2}^{r}, p\left(C_{i}^{r}\right) \leq r, i=1,2\right\} .
$$

Now, let $A=\left\{(t, 0) \in \mathbb{R}^{2}: 0 \leq t \leq 2\right\}, B=\left\{(t, 0) \in \mathbb{R}^{2}: 0 \leq t \leq 1\right\}, \epsilon>0$ be arbitrary and

$$
B_{\epsilon}=\left\{\left(x_{1}, x_{2}\right) \in \mathbb{R}^{2}: 0 \leq x_{1} \leq 1,0 \leq x_{2}<\epsilon\right\} .
$$

Then there exists $B_{\epsilon} \in V$ for every $V \in \mathcal{N}_{\theta}$ such that $A \subset B+B_{\epsilon}$. However $A \nsubseteq B$.

Remark 3.3. In Lemma 2.3, the hypothesis "Let $X$ be a normed qls" can not be relaxed. Indeed, let us recall that every linear space is a qls with the partial order relation " $=$ " and consider the element $x=\left(x_{1}, x_{2}\right)$ and the seminorm

$$
p(x)=p\left(\left(x_{1}, x_{2}\right)\right)=\left\{\left|x_{1}\right|:\left(x_{1}, x_{2}\right) \in \mathbb{R}^{2}\right\}
$$

on the qls $\left(\mathbb{R}^{2},=\right)$.

Let $\left(x_{n}\right)=\left(\left(\frac{1}{n}, 0\right)\right)_{n=1}^{\infty}$ and $\left(y_{n}\right)=\left(\left(\frac{1}{n}, 0\right)\right)_{n=1}^{\infty}$.

We see that $x_{n}=y_{n}$ for every $n$. On the other hand, the sequence $\left(\left(\frac{1}{n}, 0\right)\right)_{n=1}^{\infty}$ converges to different two elements of $\mathbb{R}^{2}$ according to this seminorm. For example,

$$
\left(x_{n}\right)=\left(\left(\frac{1}{n}, 0\right)\right)_{n=1}^{\infty} \rightarrow(0,1)=x
$$

and

$$
\left(y_{n}\right)=\left(\left(\frac{1}{n}, 0\right)\right)_{n=1}^{\infty} \rightarrow(0,2)=y
$$

since

$$
p\left(\left(\frac{1}{n}, 0\right)-(0,1)\right)=p\left(\left(\frac{1}{n},-1\right)\right)=\left|\frac{1}{n}\right| \rightarrow 0
$$

and

$$
p\left(\left(\frac{1}{n}, 0\right)-(0,2)\right)=p\left(\left(\frac{1}{n},-2\right)\right)=\left|\frac{1}{n}\right| \rightarrow 0
$$

while $n \rightarrow \infty$. However $x \neq y$.

Now, let us denote by $\Omega_{C}^{n}(\mathbb{R})$ the family of all $n$-tuples intervals which constitute an important part of interval analysis.

$$
\Omega_{C}^{n}(\mathbb{R})=\left\{X=\left(X_{1}, X_{2}, \ldots, X_{n}\right): X_{i} \in \Omega_{C}(\mathbb{R}) \text { for } 1 \leq i \leq n\right\} .
$$

We emphasize that $\Omega_{C}^{n}(\mathbb{R})$ is different from $\Omega_{C}\left(\mathbb{R}^{n}\right)$ which is the family of all nonempty closed, bounded and convex subsets of $\mathbb{R}^{n}$. 

by

$\Omega_{C}^{n}(\mathbb{R})$ is a qls with the operations " $\oplus$ ", " $\odot$ " and partial order relation " $\preceq$ " defined

$$
\begin{gathered}
\oplus: \Omega_{C}^{n}(\mathbb{R}) \times \Omega_{C}^{n}(\mathbb{R}) \rightarrow \Omega_{C}^{n}(\mathbb{R}), \\
X \oplus Y=\left(X_{1}+Y_{1}, X_{2}+Y_{2}, \ldots, X_{n}+Y_{n}\right)
\end{gathered}
$$

and

$$
\begin{gathered}
\odot: \mathbb{R} \times \Omega_{C}^{n}(\mathbb{R}) \rightarrow \Omega_{C}^{n}(\mathbb{R}), \\
\alpha \odot X=\left(\alpha \cdot X_{1}, \alpha \cdot X_{2}, \ldots, \alpha \cdot X_{n}\right)
\end{gathered}
$$

and

$$
X \preceq Y \Leftrightarrow X_{i} \subseteq Y_{i} \text { for every } i \in\{1,2, \ldots, n\}
$$

for $X=\left(X_{1}, X_{2}, \ldots, X_{n}\right), Y=\left(Y_{1}, Y_{2}, \ldots, Y_{n}\right) \in \Omega_{C}^{n}(\mathbb{R})$ and $\alpha \in \mathbb{R}$.

$\Omega_{C}^{n}(\mathbb{R})$ is a seminormed qls with equality defined by

$$
\|X\|_{\Omega_{C}^{n}(\mathbb{R})}=\left\|X_{i}\right\|_{\Omega_{C}(\mathbb{R})}
$$

for fixed $i \in\{1,2, \ldots, n\}$.

For example, on seminormed qls $\Omega_{C}^{3}(\mathbb{R})$, the equality

$$
\|X\|_{\Omega_{C}^{3}(\mathbb{R})}=\left\|X_{1}\right\|_{\Omega_{C}(\mathbb{R})}
$$

defines a seminorm. It is not hard to see that seminorm axioms are hold. The function defined by this way is not a norm since $\|X\|=0$ for element

$$
X=\{[0,0],[1,3],[-3,-2]\} \in \Omega_{C}^{3}(\mathbb{R}) \neq \theta .
$$

Example 3.4. Also the condition (2.18) is also not satisfied:

Let $X=\{[1,2],[3,5],[-4,-3]\}, Y=\{[1,2],[4,6],[3,5]\}$ and $\epsilon>0$ be arbitrary. Let us define as

$$
X_{\epsilon}=\{[0,0],[-2,1],[-8,-7]\} .
$$

Then $\left\|X_{\epsilon}\right\|=0$ and $X \preceq Y+X_{\epsilon}$, but $X \npreceq Y$.

We note that $T$ will be called as a linear operator between quasilinear spaces, if $T$ satisfies the following conditions:

$$
\begin{gathered}
T(\alpha \cdot x)=\alpha \cdot T(x) \text { for any } \alpha \in \mathbb{R}, \\
T\left(x_{1}+x_{2}\right)=T\left(x_{1}\right)+T\left(x_{2}\right) .
\end{gathered}
$$

Also, any linear operator from the quasilinear space $X$ to $\mathbb{R}$ is called a linear functional on quasilinear space $X$.

The Hahn-Banach theorem is an important tool in functional analysis and there are several versions of it. Let us note that we are largely inspired by Theorem 2.2 in [4], in stating the Hahn-Banach theorem for seminormed quasilinear spaces. The impact of the Hahn-Banach theorem is the existence of linear functionals having specified properties on a quasilinear space. The following is the main result of our work. 
Theorem 3.1. Let $p$ be a seminorm on the quasilinear space $X$ and $Y$ be a subspace of $X$. Suppose that $f$ is a linear functional from $Y$ to $\mathbb{R}$ and $f(y) \leq p(y)$ for all $y \in Y$. Suppose also that $\varphi$ is a quasilinear functional from $X$ to $\Omega_{C}(\mathbb{R})$ and $f(x) \in \varphi(x)$ for every $x \in Y$. Then there exists a linear functional $g$ from $X$ to $\mathbb{R}$ such that $g(x)=f(x)$ for any $x \in Y$ and $g(x) \in \varphi(x)$ for any $x \in X$.

Proof. Let $Z$ be a subspace of $X$ containing $Y, g$ be a linear functional on $Z$ that extends $f$, and $g(z) \leq p(z)$ for all $z \in Z$. Also, let $\mathcal{Z}$ be the set of all pairs $(Z, g)$. First of all, since the pair $(Y, f)$ is obviously an element of $\mathcal{Z}$, the set $\mathcal{Z}$ is not empty.

Define a partial order relation " $\ll$ " on $\mathcal{Z}$ as follows:

$$
\left(Z_{1}, g_{1}\right) \ll\left(Z_{2}, g_{2}\right) \Leftrightarrow\left\{\begin{array}{c}
Z_{1} \subset Z_{2} \\
g_{2}(z)=g_{1}(z), \text { for all } z \in Z_{1} .
\end{array}\right.
$$

Using Zorn Lemma, $\mathcal{Z}$ posesses a maximal totally ordered subset $\left\{\left(Z_{\alpha}, g_{\alpha}\right)\right\}$. If it is defined as $Z=\bigcup Z_{\alpha}$, clearly, $Z$ is a subspace of $X$. Also, if $z \in Z$, then $z \in Z_{\alpha}$ for some $\alpha$.

If $z \in Z_{\alpha}$ and $z \in Z_{\beta}$, then, without loss of generality, we may assume that $\left(Z_{\alpha}, g_{\alpha}\right) \ll\left(Z_{\beta}, g_{\beta}\right)$. Therefore $g_{\alpha}(z)=g_{\beta}(z)$, so that we may uniquely define $g(z)=g_{\alpha}(z)$ whenever $z \in Z_{\alpha}$.

Now, let us show that the function $g$ defined by this way is a linear functional on $Z$. To do this, let $z_{1}$ and $z_{2}$ be elements of $Z$. Then $z_{1} \in Z_{\alpha}$ and $z_{2} \in Z_{\beta}$ for some $\alpha$ and $\beta$. Since the set $\left\{\left(Z_{\gamma}, g_{\gamma}\right)\right\}$ is totally ordered, we may assume, again without loss of generality, that $Z_{\alpha} \subset Z_{\beta}$, hence both $z_{1}$ and $z_{2}$ are in $Z_{\beta}$. So

$g\left(\lambda_{1} \cdot z_{1}+\lambda_{2} \cdot z_{2}\right)=g_{\beta}\left(\lambda_{1} \cdot z_{1}+\lambda_{2} \cdot z_{2}\right)=\lambda_{1} g_{\beta}\left(z_{1}\right)+\lambda_{2} g_{\beta}\left(z_{2}\right)=\lambda_{1} g\left(z_{1}\right)+\lambda_{2} g\left(z_{2}\right)$.

We note that if $y \in Y$, then $g(y)=f(y)$, so that $g$ is an extension of $f$. So, $g$ is a linear functional on the subspace $Z$, that extends $f$, for which $g(z) \leq p(z)$ and $g(z) \in \varphi(z)$ for all $z \in Z$, so that the proof will be complete if we show that $Z=X$.

Assume that $Z \neq X$, and $v$ be an element in $X$ which is not in $Z$. Also, $Z^{\prime}$ denotes the set of all elements in the form $z+\lambda \cdot v$ for $\lambda \in \mathbb{R}$ and $z \in Z$.

On the other hand, since

$$
\theta=(1-1) \cdot v \preceq v-v
$$

and

$$
z+z^{\prime} \preceq z+z^{\prime} \text { for any } z, z^{\prime} \in Z,
$$

we write $z+z^{\prime} \preceq z+z^{\prime}+v-v$ from (2.12). Also $p\left(z+z^{\prime}\right) \leq p\left(z+z^{\prime}+v-v\right)$ by the fact that $p$ is a seminorm. Therefore, we observe

$$
\begin{aligned}
g(z)+g\left(z^{\prime}\right) & =g\left(z+z^{\prime}\right) \\
& \leq p\left(z+z^{\prime}\right) \\
& \leq p\left(z+z^{\prime}+v-v\right) \\
& \leq p(z+v)+p\left(z^{\prime}-v\right)
\end{aligned}
$$


or

$$
g\left(z^{\prime}\right)-p\left(z^{\prime}-v\right) \leq p(z+v)-g(z)
$$

for any $z, z^{\prime} \in Z$.

Consider the sets

$$
\begin{gathered}
W_{1}=\left\{g\left(z^{\prime}\right)-p\left(z^{\prime}-v\right): z^{\prime} \in Z\right\} \subset \mathbb{R}, \\
W_{2}=\{p(z+v)-g(z): z \in Z\} \subset \mathbb{R}
\end{gathered}
$$

and say

$$
\sup W_{1}=w_{1} \text { and } \inf W_{2}=w_{2} .
$$

It is clear that $w_{1} \leq w_{2}$. Take $w_{0}$ to be any number for which $w_{1} \leq w_{0} \leq w_{2}$ and define $g^{\prime}$ on $Z^{\prime}$ by

$$
g^{\prime}(z+\lambda \cdot v)=g(z)+\lambda \cdot w_{0} .
$$

It is easy to see that $g^{\prime}$ is linear and extends $f$.

If $\lambda>0$, then

$$
\begin{aligned}
g^{\prime}(z+\lambda \cdot v) & =\lambda\left(g\left(\frac{z}{\lambda}\right)+w_{0}\right) \\
& \leq \lambda\left(g\left(\frac{z}{\lambda}\right)+w_{2}\right) \\
& \leq \lambda\left(g\left(\frac{z}{\lambda}\right)+p\left(\frac{z}{\lambda}+v\right)-g\left(\frac{z}{\lambda}\right)\right) \\
& =\lambda p\left(\frac{z}{\lambda}+v\right) \\
& =p(z+\lambda \cdot v) .
\end{aligned}
$$

On the other hand, if $\lambda<0$, then

$$
\begin{aligned}
g^{\prime}(z+\lambda \cdot v) & =|\lambda|\left(g\left(\frac{z}{|\lambda|}\right)-w_{0}\right) \\
& \leq|\lambda|\left(g\left(\frac{z}{|\lambda|}\right)-w_{1}\right) \\
& \leq|\lambda|\left(g\left(\frac{z}{|\lambda|}\right)-g\left(\frac{z}{|\lambda|}\right)+p\left(\frac{z}{|\lambda|}-v\right)\right) \\
& =|\lambda| p\left(\frac{z}{|\lambda|}-v\right) \\
& =p(z+\lambda \cdot v) .
\end{aligned}
$$

This proves $g^{\prime}(z+\lambda \cdot v) \leq p(z+\lambda \cdot v)$ for all $z+\lambda \cdot v \in Z^{\prime}$. Hence $\left(Z^{\prime}, g^{\prime}\right) \in \mathcal{Z}$ and $(Z, g) \ll\left(Z^{\prime}, g^{\prime}\right)$. But then the element $\left(Z^{\prime}, g^{\prime}\right) \in \mathcal{Z}$ will contradicts with the maximality of $(Z, g)$ by the fact that $\left\{\left(Z_{\alpha}, g_{\alpha}\right)\right\}$ is a maximal totally ordered set. This completes the proof. 


\section{References}

[1] G. Alefeld, G. Mayer, Interval analysis: theory and applications, J. Comput. Appl. Math. 121 (2000) 421-464.

[2] S.M. Aseev, Quasilinear operators and their application in the theory of multivalued mappings, Proceedings of the Steklov Institute of Mathematics 2 (1986) $23-52$.

[3] J.P. Aubin, H. Frankowska, Set-Valued Analysis, Birkhäuser, Boston, 1990.

[4] L.W. Baggett, Functional Analysis: a primer, Marcel Dekker, Inc., New York, USA, 1992.

[5] S. Çakan, Y. Yılmaz, Localization Principle in Normed Quasilinear Spaces, Information Sciences and Computing, Article ID ISC530515 (2015) 15 pages.

[6] S. Çakan, Y. Yılmaz, Normed proper quasilinear spaces, J. Nonlinear Sci. Appl. 8 (2015) 816-836.

[7] S. Çakan, Y. Yılmaz, On the quasimodules and normed quasimodules, Nonlinear Funct. Anal. Appl. 20 (2) (2015) 269-288.

[8] S. Çakan, Y. Yılmaz, Lower and upper semi basis in quasilinear spaces, Erciyes University Journal of the Institute of Science and Technology 31 (2) (2015) 97104.

[9] S. Çakan, Y. Yılmaz, Lower and upper semi convergence in normed quasilinear spaces, Nonlinear Funct. Anal. Appl. 21 (3) (2016) 501-511.

[10] V. Lakshmikantham, T.G. Bhaskar, J.V. Devi, Theory of Set Differential Equations in Metric Spaces, Cambridge Scientific Publishers, Cambridge, 2006.

[11] R.E. Moore, R.B. Kearfott, M.J. Cloud, Introduction to Interval Analysis, SIAM, Philadelphia, USA, 2009.

[12] Y. Yılmaz, S. Çakan, Ş. Aytekin, Topological quasilinear spaces, Abstr. Appl. Anal., Article ID 951374 (2012) 10 pages. 
DOI: $10.7862 /$ rf.2019.5

\section{Sümeyye Çakan}

email: sumeyye.tay@gmail.com

ORCID: 0000-0001-8761-8564

Department of Mathematics

İnönü University

Malatya 44280

TURKEY

\section{Yilmaz Yilmaz}

email: yyilmaz44@gmail.com

ORCID: 0000-0002-2197-3579

Department of Mathematics

İnönü University

Malatya 44280

TURKEY

Received 14.11.2018 